Aim(s)/objectives To assess the background, demographics, presentation and follow up of patients with confirmed LGV infection in an inner London cohort. To analyse compliance with BASHH auditable measures surrounding follow up testing including HIV and hepatitis C.

Methods A retrospective case note review was conducted of all PCR confirmed LGV infections from 01.01.2005-31.07.14. Data was extracted looking at the demographics, presentation, risk factors, concurrent STIs and follow up of patients as per BASHH audit standards.

Results 44 patients were identified. 43 were MSM and 1 a heterosexual female with a bisexual partner. $80 \%$ (35) presented with symptomatic LGV infection and 20\% (9) had had a previous infection with LGV. 43\% (19) were diagnosed with concurrent STIs; of which only 4 had extra rectal chlamydia (3 urethral and 1 eye). 64\% (28) were known to be HIV positive at LGV diagnosis. Only 69\% (11) of the remaining HIV negative patients had a documented HIV follow up test within 12 months of LGV diagnoses. 36\% (4) of these were newly diagnosed with HIV. Out of the 24 documented hepatitis $\mathrm{C}$ tests within 12 months of LGV diagnosis there were 2 new cases of hepatitis C.

Discussion/conclusion Our cohort largely reflects the UK epidemic and reinforces the strong association with HIV infection. The audit reveals poor adherence to BASHH standards for repeat testing, which will be addressed with a specific active recall process.

\section{P17 ENHANCED SEXUAL HEALTH SERVICES IN COMMUNITY PHARMACIES - PILOT}

Anthony Peacham*, Merle Symonds. Barts Health NHS Trust, London, UK

10.1136/sextrans-2015-052126.61

Background/introduction STI screening via community pharmacies (CPs) has traditionally been very low.

Aim(s)/objectives To increase STI screening in young people (15-24 years) in a London Borough with high rates of infection using a new self-test kit (testing for Chlamydia and Gonorrhoea (CT/GC), and HIV) alongside condom distribution via the panLondon condom scheme.

Methods Nine CPs were selected based on high rates of Emergency Contraception provision and condom distribution in 2013. Frontline staff were trained and care pathways established. Ongoing monthly support was provided by site-visit and phone. Results were notified by text. Positive results, partner notification and follow-up were managed by a Level 3 GUM clinic. Evaluation was by user/CP survey.

Results

- 8 CPs were active during the pilot which ran January-December 2014.

- 214 self-test kits were distributed; $108 \mathrm{CT} / \mathrm{GC}$ tests and 96 HIV tests were returned/tested (return rates of $50.5 \%$ and 44.9\% respectively). At the start $1 \mathrm{CP}$ removed HIV tests from packs.

- 4,476 condoms were distributed.

- 7 Chlamydia positives were identified (positivity 6.5\%).

- Quarter 12014 saw a 700\% increase in numbers of STI tests processed) in the 9 CPs compared to Quarter 32013 (prepilot levels). This significantly increased activity continued throughout 2014.

- All users were very or quite satisfied with the service and were very or quite likely to use the service again.

- $66 \%$ were very likely to recommend the service to others
Discussion/conclusion With adequate training and support, community pharmacies provide an engaged, accessible and convenient venue for STI testing (including HIV) and condom distribution.

\section{P18 EXPLORING QUANTITATIVE RELATIONSHIPS BETWEEN SEROLOGICAL RESULTS AND STAGE OF SYPHILIS}

Johanna Denman*, Imali Fernando. NHS Lothian, Edinburgh, UK

10.1136/sextrans-2015-052126.62

Background/introduction There has been little published regarding quantitative results of newer serological assays in infectious syphilis. Previous studies have shown an association between VDRL and TPPA titre and stage of syphilis; with higher titres in secondary syphilis.

Aim(s)/objectives To examine quantitative relationships between serological results and stage of syphilis including newer assays.

Methods Early syphilis cases diagnosed March 2011-August 2014 were identified from a sexual health clinic database. Cases classified as primary, secondary and early latent by clinical diagnosis. Serology results were recorded including TPPA, VDRL (used until 01/03/2012), RPR (used from 01/03/2012), IgG (Abbott Architect Total Antibody Test), and IgM (lab21 IgM EIA).

Results 155 patients included. 149 male, 6 female. Average age 38. $92 \%$ men were MSM. 32\% HIV positive. $33 \%$ classified as primary, $21 \%$ secondary, $46 \%$ early latent. $64 \%$ new diagnoses, $36 \%$ re-infected.

\begin{tabular}{llllll}
\multicolumn{7}{c}{ Abstract P18 Table 1} & Syphilis stage and serology & \\
\hline \multicolumn{7}{c}{ RPR } & VDRL & TPPA & & \\
& $(\mathrm{n}=110)$ & $(\mathrm{n}=45)$ & $(\mathrm{n}=155)$ & IgM & IgG \\
& Median & Median & Median & $(\mathrm{n}=122)$ & $(\mathrm{n}=155)$ \\
& $($ range $)$ & $($ range $)$ & $($ range $)$ & Mean & Mean \\
\hline Primary & $4(0-64)$ & $4(0-64)$ & $2560(0->5120)$ & 4.06 & 20.44 \\
Secondary & $32(4-128)$ & $64(16-256)$ & $>5120(2560->5120)$ & 10.09 & 37.28 \\
Early Latent & $8(0-128)$ & $16(0-512)$ & $5120(40->5120)$ & 3.84 & 29.58 \\
\hline
\end{tabular}

Discussion/conclusion Results confirmed the quantitative relationship between syphilis stage and VDRL and TPPA titre identified previously. Additionally this study showed that IgM and IgG values, using Lab 21 IgM EIA and Abbott Architect Total Antibody Test assays, are also linked to stage of syphilis. Unsurprisingly, IgG titres were highest in secondary and lowest in primary syphilis. IgM values were lowest in early latent and highest in secondary syphilis.

\section{P19 MEASURING THE IMPACT OF SUPPLEMENTARY TESTING OF NEISSERIA GONORRHOEA POSITIVE NUCLEIC ACID AMPLIFICATION TESTS ON THE RATE OF EXTRA- GENITAL NEISSERIA GONORRHOEA DIAGNOSIS AND CONCORDANCE OF NAATS WITH BACTERIAL CULTURE}

Rachel Coyle*, Michael Rayment, Sarah Creighton. Homerton University Hospital, London, UK

\subsection{6/sextrans-2015-052126.63}

Background/introduction Nucleic-acid amplification tests (NAATs) are more sensitive in the detection of Neisseria Gonorrhoea (NG) 
than culture or microscopy, but specificity at extra-genital sites may be lower due to cross reactivity with other Neisseria species. BASHH recommends supplementary testing of NG positive extragenital NAATs to improve specificity. This inner city DGH introduced supplementary testing on 01/11/13.

Aim(s)/objectives To evaluate the impact of introducing supplementary testing on the rate of extra-genital NG diagnosis and concordance of positive NAATs with culture.

Methods All patients with a diagnosis of NG at any site between 01/08/13 and 31/01/14 were identified. Concordance of positive NAATs with bacterial culture pre- and post-intervention was reviewed.

Results There were 471 positive NAATs from 372 patients during the study period. Extra-genital samples accounted for $48.6 \%(\mathrm{n}=118 / 243)$ of positive NAATS pre-intervention and $41.2 \%(\mathrm{n}=94 / 228)$ post-intervention, $(\mathrm{p}=0.03)$. Culture was obtained from 305 sites, 119 of which were extra-genital. Concordance pre- and post-intervention is detailed in below.

\begin{tabular}{llll}
\hline Abstract P19 & Table 1 & Gonorrhoea test concordance \\
\hline & \multicolumn{3}{c}{ Proportion of cultures positive for NG } \\
\cline { 2 - 4 } & Pre intervention & Post intervention & $P$ value \\
\hline Rectal & $9 / 21(42.8 \%)$ & $10 / 19(53.0 \%)$ & 0.39 \\
Pharyngeal & $3 / 49(6.10 \%)$ & $3 / 31(9.67 \%)$ & 0.40 \\
\hline
\end{tabular}

Discussion/conclusion The proportion of NG positive NAATs from extra-genital sites fell following the introduction of supplementary testing, which may reflect an improvement in specificity. A non-significant increase in concordance of NAATs with culture was noted however concordance was low overall, especially in the pharynx. It remains unclear whether discordant results represent lower sensitivity of culture, lower specificity NAATs despite supplementary testing, or spontaneous clearance between screening and recall for culture.

\section{P20 INVESTIGATION INTO AN INCREASE OF DIAGNOSES OF GONORRHOEA IN SOUTHEND-ON-SEA}

${ }^{1}$ Lynsey Emmett* ${ }^{2}$ Henna Jaleel, ${ }^{2}$ Mohd Sabri Abu Bakar, ${ }^{2}$ Donna Stookes, ${ }^{3}$ Karen Payne, ${ }^{4}$ Simon Ford, 'Mark Reacher, 'Sultan Salimee. 'Public Health England, East, UK; ${ }^{2}$ Southend University Hospital NHS Foundation Trust, Southend-on-Sea, UK; ${ }^{3}$ South Essex Partnership University NHS Foundation Trust, Southend-on-Sea, UK; ${ }^{4}$ Southend-onSea Borough Council, Southend-on-Sea, UK

\subsection{6/sextrans-2015-052126.64}

Background/introduction Between 2012 and 2013 the rate of gonorrhoea in Southend increased significantly from 24.6 to 42.4 per 100,000 . A multidisciplinary Incident Management Team was established in June 2014 to assess the situation and implement appropriate control measures. However, the number of cases had already begun to fall. A retrospective case review was initiated.

Aim(s)/objectives To identify factors that contributed to the increase and subsequent decrease in diagnoses.

Methods Enhanced questionnaires were completed for each case diagnosed between October 2012 and March 2014. Antibiotic resistance profiles were provided by the local laboratory.
Previous STI and HIV test history was extracted from the Genitourinary Medicine Clinic Activity Dataset (GUMCADv2).

Results Provisional results show that enhanced forms were completed for 160 cases. Majority of cases were of white ethnicity $(64 \%)$ and born in the UK (87\%). Cases were aged between 15 to 63 years (median 28 years), 62\% were male and 60\% heterosexual. Most cases had 1 or 2 partners in the preceding 3 months and attended because of symptoms (40\%). However, approximately 30 cases had been referred from a level 2 service - some of which had negative results when re-tested. The majority of cases were treated with first line therapy and had a test of cure undertaken.

Discussion/conclusion Full details of the epidemiology, presentation and diagnosis of the cases will be presented - including a comparison with the cases diagnosed before and after the increase, the use of social network techniques and an analysis against the auditable outcome measures in the BASHH guidelines.

\section{P21 IS IT SYPHILIS? THE DARK ART OF INTERPRETING SYPHILIS SEROLOGY}

Hemanti Patel, Sarah Alexander, Helen Fifer*. Public Health England, London, UK

\subsection{6/sextrans-2015-052126.65}

Background/introduction The diagnosis of syphilis relies mainly upon a panel of serological tests. A sensitive treponemal test such as the enzyme immunoassay (EIA) is used as a screening test; another treponemal test such as the Treponema pallidum particle agglutination (TPPA) assay is used to confirm a reactive screening test. Difficulties arise when these tests produce a discordant result. Our laboratory uses the INNO-LIA immunoblot assay to resolve discordant screening results.

Aim(s)/objectives To evaluate whether the use of the INNO-LIA enables clinically useful interpretation.

Methods We reviewed the last 100 INNO-LIA tests performed by our laboratory.

Results Comparison of EIA, TPPA and INNO-LIA results are shown in Table 1. The Antibody Index is a measure of the positive signal in the EIA ( $\geq 1.2$ is a positive result).

Discussion/conclusion In EIA positive (AI $\geq 5$ )/TPPA equivocal cases the INNO-LIA was always positive or equivocal, consistent with treponemal infection. The INNO-LIA test may be unnecessary in these cases. In EIA positive/TPPA negative cases, the INNO-LIA is able to resolve the discordant result less than half of the time. Overall the INNO-LIA produced equivocal results in $44 \%$ of serums, which is unsatisfactory for confirming the diagnosis of syphilis. Although the INNO-LIA does help resolve some cases, there remains a need for new diagnostics.

\begin{tabular}{|c|c|c|c|c|c|c|c|}
\hline \multirow{2}{*}{$\begin{array}{l}\text { No. of } \\
\text { samples }\end{array}$} & \multirow[b]{2}{*}{ EIA } & \multirow{2}{*}{$\begin{array}{l}\text { EIA } \\
\text { Antibody } \\
\text { Index (Al) }\end{array}$} & \multirow[b]{2}{*}{ TPPA } & \multicolumn{3}{|c|}{ INNO-LIA } & \multirow{2}{*}{$\begin{array}{l}\text { Discordan } \\
\text { result } \\
\text { resolved? }\end{array}$} \\
\hline & & & & Negative & Positive & Equivocal & \\
\hline 41 & Positive & $\geq 5$ & Negative & 5 & 12 & 24 & $41 \%$ \\
\hline 30 & Positive & $\geq 5$ & Equivocal & 0 & 20 & 10 & $100 \%$ \\
\hline 26 & Positive & $\leq 5$ & Equivocal & 5 & 13 & 8 & $81 \%$ \\
\hline 3 & Positive & $\leq 5$ & Negative & 1 & 0 & 2 & $33 \%$ \\
\hline
\end{tabular}

